\title{
Acoustic-gravity waves in the atmosphere generated by infragravity waves in the ocean
}

\author{
Oleg A Godin ${ }^{1,2^{*}}$, Nikolay A Zabotin ${ }^{1,3}$ and Terence W Bullett ${ }^{1,4}$
}

\begin{abstract}
Infragravity waves are surface gravity waves in the ocean with periods longer than approximately $30 \mathrm{~s}$. Infragravity waves propagate transoceanic distances and, because of their long wavelengths, provide a mechanism for coupling wave processes in the ocean, atmosphere, and the solid Earth. Here, we present a strict physical justification for the hypothesis that background ocean waves may generate waves in the upper atmosphere. We show that, at frequencies below a certain transition frequency of about $3 \mathrm{mHz}$, infragravity waves continuously radiate their energy into the upper atmosphere in the form of acoustic-gravity waves. Based on ionospheric observations and estimates of the fluxes of the mechanical energy and momentum from the deep ocean, we conclude that acoustic-gravity waves of oceanic origin may have an observable impact on the upper atmosphere. We anticipate our work to be a starting point for a detailed analysis of global manifestations of the ocean-generated background acoustic-gravity waves.
\end{abstract}

Keywords: Acoustic-gravity waves; Infragravity waves; Air-sea interaction; lonosphere; Radio sounding

\section{Findings}

\section{Introduction}

Ionospheric manifestations of tsunamis (Artru et al. 2005; Makela et al. 2011; Galvan et al. 2012; Komjathy et al. 2012; Occhipinti et al. 2013) provided dramatic evidence of coupling between wave processes in the ocean and in the atmosphere. But tsunamis are only a transient feature of a more general phenomenon: infragravity waves (IGWs) (Webb et al. 1991; Herbers et al. 1995; Aucan and Ardhuin 2013; Godin et al. 2014). IGWs are permanently present surface gravity waves in the ocean with periods longer than the longest periods (approximately $30 \mathrm{~s}$ ) of wind-generated waves. IGWs propagate transoceanic distances and, because of their long wavelengths (from approximately $1 \mathrm{~km}$ to hundreds of $\mathrm{km}$ ), offer a mechanism for coupling wave processes in the ocean, atmosphere, and the solid Earth (Hines 1972; Crawford et al. 1998; Rhie and Romanowicz 2004; Bromirski and Stephen 2012). The notion that background ocean waves may generate waves in the atmosphere has existed for a long time (Hines 1972; Peltier and Hines 1976; Livneh et al. 2007),

\footnotetext{
* Correspondence: Oleg.Godin@colorado.edu

${ }^{1}$ Cooperative Institute for Research in Environmental Sciences, University of

Colorado, Boulder, CO 80309, USA

${ }^{2}$ Physical Sciences Division, NOAA Earth System Research Laboratory,

Boulder, CO 80305, USA

Full list of author information is available at the end of the article
}

but no quantitative theory of this effect has been suggested. Here, we provide a strict physical justification for this hypothesis. Using a coupled atmosphere-ocean model taking into account both compressibility of the air and the gravity, we show that there exist two distinct regimes of IGW penetration into the atmosphere. At frequencies above a transition frequency, there are evanescent waves in the atmosphere propagating horizontally along the ocean surface. At frequencies below the transition frequency, IGWs continuously radiate their energy into the upper atmosphere in the form of acoustic-gravity waves (AGWs). The transition frequency is a function of ocean depth; it varies slowly near $3 \mathrm{mHz}$ for typical depth values and drops sharply to zero only for extreme depth values. Using a semi-empirical model of the IGW power spectrum (Godin et al. 2013), we derive an estimate of the flux of the mechanical energy and mechanical momentum from the deep ocean into the atmosphere due to IGWs and predict specific forcing on the atmosphere in coastal regions. We conclude that AGWs of oceanic origin may have an observable impact on the upper atmosphere.

\section{Theory \\ Infragravity waves and atmospheric waves \\ IGWs are usually modeled as surface gravity waves in an incompressible ocean with a free surface and a rigid}

\section{穴}


seafloor. In this approximation, IGWs have the dispersion equation

$$
\omega^{2}=g k \tan h k H,
$$

where $\omega$ and $k$ are the wave frequency and wavenumber, $g \approx 9.8 \mathrm{~m} / \mathrm{s}^{2}$ is the acceleration due to gravity, and $H$ is the ocean depth (Gill 1982). IGW phase speed $c=\omega / k$ does not exceed $(g H)^{1 / 2}$ and is much smaller than sound speed in water and compressional and shear wave speeds in the ocean bottom. Therefore, corrections to the dispersion equation due to water compressibility and seafloor compliance are rather small (Tsai et al. 2013; Watada et al. 2014) and do not change the nature of IGWs as horizontally propagating surface waves, where both $k$ and $\omega$ are real-valued (Brekhovskikh and Godin 1998).

The free-surface boundary condition implies that the wave-induced motion of the air has no effect on oceanic waves. For a simple description of coupling between waves in the ocean and atmosphere, we model the latter as an isothermal gas half-space. Continuous waves with horizontal wave vector $\mathbf{k}$ on the sea surface generate plane waves in the half-space. Horizontal and vertical components of the wave vector of the atmospheric waves are $\mathbf{k}$ and $m$, where

$$
m^{2}=\frac{\omega^{2}}{c_{s}^{2}}-k^{2}-\frac{\gamma^{2} g^{2}}{4 c_{s}^{4}}+\frac{(\gamma-1) g^{2} k^{2}}{\omega^{2} c_{s}^{2}},
$$

$k^{2}=\mathbf{k} \cdot \mathbf{k}, c_{s}$ is the sound speed in air, and $\gamma \approx 1.4$ is the ratio of specific heats at constant pressure and constant volume (Gill 1982). The atmospheric waves have a finite impedance, i.e., the ratio of pressure perturbation in a fluid parcel to its vertical velocity. All these quantities must be continuous on the air-sea interface (Gill 1982). For oceanic waves, the impedance is small as long as the ratio $R \sim 1.3 \times 10^{-3}$ of the mass densities of the air and water at the water-air interface is small. We use a perturbation theory to quantify the coupling between oceanic and atmospheric waves.

\section{Perturbation theory}

Our theoretical analysis of the effects of the atmospheric load on infragravity waves is based on modeling the ocean surface as a boundary with finite impedance and application of the recently developed perturbation theory (Godin 2012) for normal modes of acoustic-gravity waves. The perturbation theory predicts the change in the dispersion relation of normal modes of acousticgravity waves in generic waveguides in horizontally stratified fluids, which results from small perturbations of the waveguide's parameters.

Introduce a Cartesian coordinate system with horizontal coordinates $x$ and $y$ and a vertical coordinate $z$ increasing upwards. Consider a fluid layer with a rigid boundary at $z=-H$ and a compliant boundary that coincides with the horizontal plane $z=0$ in the absence of waves. The fluid is stationary in the absence of waves. Let $W_{0}(z, \omega) \exp (i k(\omega) x-i \omega t)$ and $P_{0}(z, \omega) \exp (i k(\omega) x-i \omega t)$ describe the wave-induced vertical displacement of fluid parcels and pressure variation, which satisfy linearized (with respect to the wave amplitude) governing equations and boundary conditions $W(z, \omega)=0, z=-H$ on the rigid boundary and $P(z, \omega)=\rho g W(z, \omega), z=0$ on the free surface (Gill 1982). Here, $t$ is time, $\omega$ is wave frequency, $k$ is wavenumber, and $\rho$ is fluid density. The functions $W_{0}(z, \omega)$ and $P_{0}(z, \omega)$ describe a normal mode with the dispersion relation $k(\omega)$ in an unperturbed waveguide and are assumed to be known.

Now, consider a waveguide that differs from an unperturbed waveguide by having a different upper boundary. For waves with harmonic dependence $\exp (i k x-i \omega t)$ on horizontal coordinates and time, the boundary condition can be written as

$$
\begin{aligned}
P(z, \omega)-\rho g W(z, \omega) & =-i \omega Z(k, \omega) W(z, \omega), \\
z & =0 .
\end{aligned}
$$

Here, $P-\rho g W$ and $Z$ have the physical meaning, respectively, of the wave-induced pressure variation in a moving fluid parcel (i.e., Lagrangian pressure perturbation) and the boundary impedance (Godin 2012). Impedance of the free boundary equals zero. When $Z$ is small, there exists a normal mode with the dispersion relation $k_{1}(\omega)=k(\omega)+\delta k(\omega)($ Godin 2012), where

$$
\begin{gathered}
\delta k=\frac{i \omega^{3}}{2 k} W_{0}^{2}(0, \omega)\left[\int_{-H}^{0} \frac{d z}{\rho} P_{0}^{2}(z, \omega)\right]^{-1} \\
Z(k(\omega), \omega)+O\left(Z^{2}\right) .
\end{gathered}
$$

Note that the impedance in the right-hand side of Equation (4) is calculated for the unperturbed value of the normal mode wavenumber.

IGWs are usually modeled as surface gravity waves in an incompressible ocean with a constant water density, a free surface, and a rigid seafloor. In this approximation, one has (Gill 1982)

$$
\begin{aligned}
P(z, \omega) & =P(0, \omega) \frac{\cosh k_{0}(z+H)}{\cosh k_{0} H}, \\
W(z, \omega) & =\frac{P(0, \omega)}{\rho g} \frac{\sinh k_{0}(z+H)}{\sinh k_{0} H},
\end{aligned}
$$

and the unperturbed dispersion relation (1).

Lagrangian pressure perturbations and normal displacement are continuous at fluid-fluid interfaces (Gill 1982). Therefore, the impedance $Z(k, \omega)$ is also continuous and can be calculated from the ratio of the 
Lagrangian pressure perturbation and the vertical displacement in an atmospheric wave with the frequency $\omega$ and horizontal wavenumber $k$. The ocean surface impedance depends on atmospheric conditions. The simplest atmospheric model is that of a stationary, isothermal, perfect gas of constant composition occupying the halfspace $z>0$. In the isothermal atmosphere, acousticgravity waves (AGWs) have the dispersion relation (2). The lower boundary of the isothermal gas half-space has the impedance (Godin 2012; Godin and Fuks 2012)

$$
Z(k, \omega)=\frac{R \rho(0)}{\omega\left(\omega^{2} c_{s}^{-2}-k^{2}\right)}\left[\omega^{2} m(k, \omega)+i g\left(k^{2}-0.5 \omega^{2} \gamma c_{s}^{-2}\right)\right],
$$

where $\rho(0)$ and $R \sim 1.3 \times 10^{-3}$ are the water density and the ratio of the air and water densities at the ocean surface. In Equation (6), the sign of the vertical component $m$ $(k, \omega)$ of the wave vector should be chosen as follows: $m=$ $i|m|$, when $m^{2}<0 ; \quad m=|m| \operatorname{sgn}\left(\omega^{2}-(\gamma-1) g^{2} c_{s}^{-2}\right)$, when $m^{2} \geq 0$ (Godin and Fuks 2012).

From Equations (1) and (4) to (6), we find the perturbation to the IGW dispersion relation (1) caused by the atmospheric loading:

$$
\delta k=\frac{R k\left(2 k^{2} c_{s}^{2}-\gamma \omega^{2}-2 i \omega^{2} c_{s}^{2} m / g\right) \sinh 2 k H}{2\left(k^{2} c_{s}^{2}-\omega^{2}\right)(\sinh 2 k H+2 k H)} .
$$

Here, only terms of the first order in $R$ are retained, and $\omega$ and $k$ satisfy the unperturbed dispersion relation (1). Equation (7) reduces to known results in the previously considered case of an infinitely deep ocean (Godin and Fuks 2012).

An equivalent of Equation (7) can be obtained, although with much more effort, without using the perturbation theory and by deriving instead an exact dispersion equation for acoustic-gravity waves in a coupled system consisting of a liquid layer and an isothermal gas half-space.

Equation (7) indicates that there are two distinct wave-propagation regimes, which are separated by a transition frequency such that $m=0$. When $m^{2}<0$ in Equation (2), waves in the air are evanescent, their energy density decreasing with height $z$ above the ocean surface as $\exp (-2 z|m|)$. Then, correction $\delta k(7)$ is real, and the IGW remains a true surface wave. When $m^{2}>0$, waves in air propagate away from the ocean surface and carry energy and momentum into the upper atmosphere. For propagating waves, the amplitude of the velocity oscillations increases with height as $\left[\rho_{a}(0) / \rho_{a}(z)\right]^{1 / 2}$, where $\rho_{a}$ $(z)$ is the air density and $\rho_{a}(0)$ refers to the mean sea level. Since the IGW phase speed is always smaller than sound speed in air, $m^{2}(2)$ can be positive only at frequencies $\omega$ smaller than the buoyancy frequency $N=(\gamma-1)^{1 / 2} g / c_{s}$. At
$\omega<N$, it follows from the radiation condition at $z \rightarrow+\infty$ that $m<0$ when $m^{2}>0$ (Godin and Fuks 2012). Then, $\delta k$ (7) is complex, and the imaginary part $\operatorname{Im} \delta k>0$. This means that, due to the atmospheric loading, the IGW slowly attenuates in the direction of its horizontal propagation and thus becomes a leaky wave (Brekhovskikh and Godin 1998). This is a natural corollary of the energy conservation and radiation of IGW energy into the atmosphere. Radiation into the atmosphere is only one of the mechanisms of IGW decay. As discussed in the section 'IGW attenuation' below, comparison with observed tsunami decay rates (Rabinovich et al. 2013) shows that the decay rate predicted by Equation (7) is smaller than measured decay rates.

\section{Radiation of atmospheric waves}

An analysis of Equations (1) and (2) shows that, within our simple environmental model, radiation of IGW energy into the atmosphere occurs at frequencies $0<\omega<\omega_{b}(H)$, where the transition frequency $\omega_{b}$ steadily decreases with depth from $\omega_{b}(0)=N$ in shallow water (about $3.0 \mathrm{mHz}$ for $\left.c_{s}=330 \mathrm{~m} / \mathrm{s}\right)$ to zero at $H=H_{b} \equiv 4(\gamma-1) \gamma^{-2} c_{s}^{2} / g \approx 0.82 c_{s}^{2} / g$ (about 9,100 $\mathrm{m}$ for $c_{s}=330 \mathrm{~m} / \mathrm{s}$; see Figure 1a). When a more realistic environmental model is used, $\omega_{b}(H)$ and $H_{b}$ also depend on the temperature and windvelocity profiles in the atmosphere. For most of the ocean, the transition frequency is between 2.5 and 3 $\mathrm{mHz}$ (Figure 1a). Only small areas of the ocean with the greatest depths, such as the Mariana, Kermadec, and Puerto Rico trenches, have transition frequencies below $2.25 \mathrm{mHz}$.

Prediction of the upper cutoff frequency $\omega_{b}$ is consistent with an earlier analysis (Peltier and Hines 1976) of the limiting case of non-dispersive tsunami waves, where $k H \ll 1$. Indirect experimental support for our predictions is provided by the observations at the Infrasound Laboratory on the Island of Hawaii that the noise level, i.e., the spectral level of ambient pressure fluctuations in air, increases sharply at frequencies below approximately $4 \mathrm{mHz}$ (Garces et al. 2011).

When investigating the conditions for IGW radiation into the atmosphere, it is imperative to consider the atmospheric waves as acoustic-gravity waves, i.e., to take into account effects due to buoyancy and compressibility simultaneously. When surface waves are considered as a potential motion of an incompressible fluid, which is an excellent approximation for wind seas (Gill 1982), orbital velocities in the wave exponentially decrease with distance from the sea surface at the same rate in the water and the air, and no radiation of wave energy into the atmosphere is predicted. In the opposite case, when buoyancy is neglected, we have acoustic waves in air, and radiation into the atmosphere occurs when the phase speed $c$ of surface 

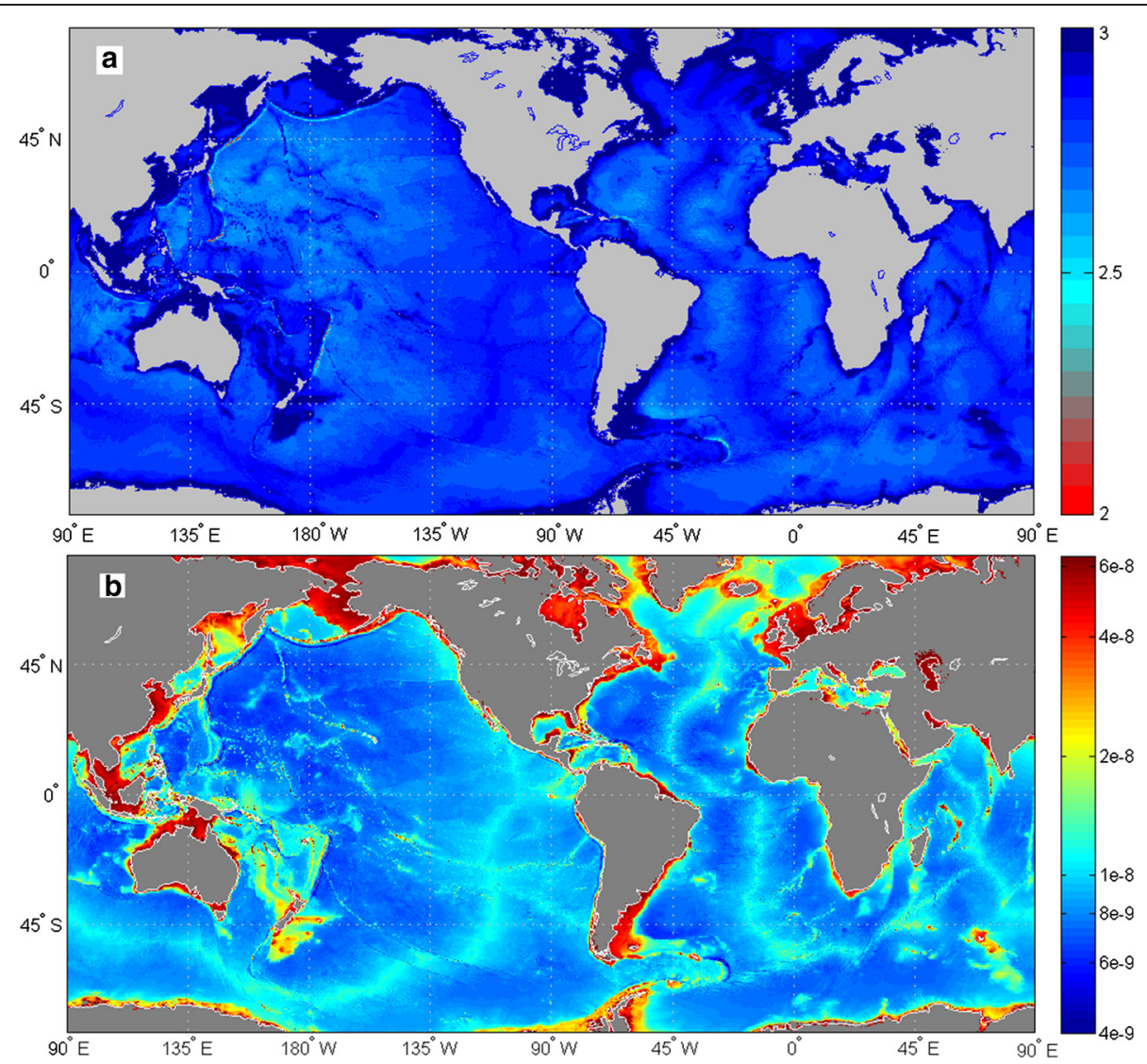

Figure 1 Geography of atmospheric wave radiation by infragravity waves in the ocean. (a) The transition frequency $\omega_{b}$ from surface to leaky infragravity waves (in $\mathrm{mHz}$ ) is shown by color for various geographical locations. At frequencies below the transition frequency, infragravity waves continuously radiate acoustic-gravity waves into the upper atmosphere. (b) The surface density / of the vertical flux of mechanical power radiated into the atmosphere (in $\mathrm{W} / \mathrm{m}^{2}$ ) is shown by color on the logarithmic scale. The transition frequency and the power flux density are calculated for an isothermal atmosphere with sound speed $c_{s}=330 \mathrm{~m} / \mathrm{s}$.

waves exceeds the sound speed (Brekhovskikh and Godin 1998). That would require the ocean depth $H>c_{s}^{2} / g$ (Eckart 1953). Perhaps surprisingly, an account of the buoyancy effects results in converse dependence on the ocean depth. As discussed above, there is no radiation into the atmosphere from a horizontally homogeneous ocean with depth $H>H_{b} \approx 0.82 c_{s}^{2} / g$. The reason for the qualitatively different behavior of acoustic and acoustic-gravity waves lies in the fact that $m^{2}$ decreases with increasing $k^{2}$, when $g=0$, but increases when $g \neq 0$ and $\omega<N$, see Equation (2).

Observations of ionospheric manifestations of recent catastrophic tsunamis (Artru et al. 2005; Makela et al. 2011; Galvan et al. 2012) serve as a spectacular manifestation of radiation of acoustic-gravity atmospheric waves by IGWs in the ocean. Background IGWs, which are believed to be generated by the nonlinear interaction of sea swell with bathymetry close to shore, have much smaller amplitude than destructive tsunamis but are continuously present throughout the ocean (Webb et al. 1991; Herbers et al. 1995; Aucan and Ardhuin 2013; Godin et al. 2014). Over extended time periods, the contribution of the background IGWs into the flux of mechanical energy from the ocean into the upper atmosphere is expected to dominate over the contributions of tsunamis. For a rough estimate, we assume that powerful tsunamis with ocean surface displacement of $0.2 \mathrm{~m}$ account for half of the tsunami energy, occur on average every 5 years, last $2 \mathrm{~h}$ at open ocean locations, and affect $10 \%$ of the ocean surface. For RMS surface elevations in background IGWs, we conservatively take $3 \mathrm{~mm}$ (Webb et al. 1991; Aucan and Ardhuin 2013; Godin et al. 2013). Then, the time-averaged tsunami energy is about $2 \%$ of the background IGW energy.

The mechanical power radiated into the atmosphere can be calculated as an integral over the vertical component of the power flux density on the ocean surface (Godin and Fuks 2012). Random IGWs with power 
spectrum $S$ of the ocean surface displacement radiate into the upper atmosphere the power

$$
I=\rho_{a}(0) \int_{\omega_{\min }}^{\omega_{b}(H)} \frac{\omega^{3} S(\omega, H)|m(\omega)| d \omega}{m^{2}(\omega)-\omega^{2} c_{s}^{-2}}
$$

per unit area of the ocean surface. Here $\omega_{\min }$ is the lowest frequency of IGWs present and $\rho_{a}(0)$ is the air density at sea level; $I=0$, when $\omega_{\min } \geq \omega_{b}(H)$. When AGWs dissipate in the upper atmosphere, they exert a force on the mean flow (wind), which can be calculated as the vertical flux of the AGW pseudomomentum (Fritts and Alexander 2003). AGW forcing plays an important role in large-scale circulation of the middle and upper atmosphere (Fritts and Alexander 2003; Geller et al. 2013). The horizontal force per unit area of the ocean surface is given by the same integral as in Equation (8) but with $\omega^{2}\langle\mathbf{k}(\omega)\rangle$ substituted for $\omega^{3}$ in the numerator in the integrand. Here, $\langle\mathbf{k}(\omega)\rangle$ is the horizontal wave vector of IGWs averaged over all propagation directions. The horizontal force is non-zero only for anisotropic IGW fields. The magnitude of the horizontal force reaches its upper bound, when all IGWs propagate in the same direction; then, $|\langle\mathbf{k}(\omega)\rangle|$ equals the wavenumber $k(\omega, H)$ of IGWs from the dispersion equation (1). This limiting case can be thought of as corresponding to IGWs generated near and propagating away from a straight shoreline. A force of the same order of magnitude in the direction perpendicular to the shoreline is expected to be applied to the upper atmosphere above the shore due to AGW radiation by IGWs with generic directivity. In the latter case, the requisite anisotropy arises from the absence of IGWs on the shore side of the coastline.

We apply the recently developed semi-empirical model $S(\omega, H)=B \tanh ^{-3 / 2} k H$, where $B=2.1 \times 10^{-5} \mathrm{~m}^{2} / \mathrm{Hz}$, of the IGW power spectrum (Godin et al. 2013) to estimate the mechanical energy and momentum radiated by the ocean into the upper atmosphere. The spectrum was obtained for IGWs with frequencies $0.3 \mathrm{mHz}$ and higher. The efficiency of AGW radiation decreases rapidly at frequencies below approximately $0.5 \mathrm{mHz}$ (Figure 2a,b), which makes the surface densities of radiated power [Equation (8)] and pseudomomentum insensitive to unknown IGW spectral levels below $0.3 \mathrm{mHz}$. The IGW spectrum and the IGW and AGW dispersion relations (1) to (7) combine to make AGW radiation more efficient at shallower ocean depths and at frequencies close to but smaller than the transition frequency $\omega_{b}$ (Figure 2a,b). The effect of the ocean depth is particularly pronounced for the momentum flux. Atmospheric waves have the unusual property that the horizontal and vertical components of their group velocity depend on frequency in rather different ways. The horizontal component is insensitive to frequency. In contrast, the vertical component (Figure 2c) and the direction of wave propagation (Figure 2d) vary strongly with frequency. The maximum vertical velocity exceeds $80 \mathrm{~m} / \mathrm{s}$ and is attained at frequencies close to 0.8 $\omega_{b}$ (Figure 2c). Geographical distribution of the mechanical energy radiation is illustrated by Figure 1b. The total power radiated into the middle and upper atmosphere by IGWs globally is estimated to be approximately $0.3 \mathrm{MW}$. The
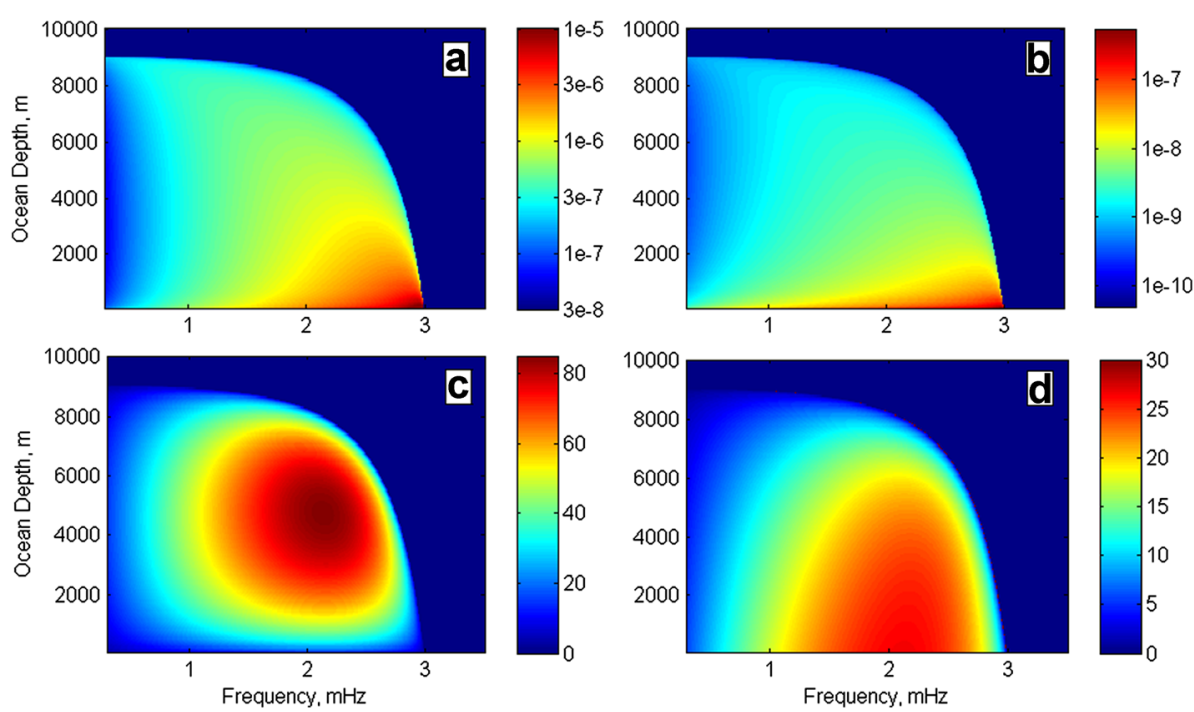

Figure 2 Spectra of atmospheric waves radiated by infragravity waves in the ocean. (a) Power spectrum of the surface density of the mechanical energy flux is shown on the logarithmic scale relative $1 \mathrm{~W} / \mathrm{m}^{2} \mathrm{~Hz}$. (b) Power spectrum of the vertical flux of horizontal pseudomomentum is shown for the straight coast model on the logarithmic scale relative $1 \mathrm{~Pa} / \mathrm{Hz}$. AGW power spectra are calculated for various ocean depths using a semi-empirical model (Godin et al. 2013) of the IGW power spectrum. (c) Vertical component of the group velocity of AGWs in m/s. (d) Angle (in degrees) between the ocean surface and the direction of AGW propagation. 
sound speed value $c_{s}=330 \mathrm{~m} / \mathrm{s}$, which is used in our model calculations, corresponds to air temperature of about $271 \mathrm{~K}$. The modeling results presented in Figures 1, 2, 3, and 4 are insensitive to the particular choice of $c_{s}$ within the range of possible sound speed values above the ocean surface.

\section{IGW attenuation}

Atmospheric loading results in the perturbation $\delta k$, which is given by Equation (7), to the dispersion relation (1) of IGWs in an ocean with a free surface. From Equation (7) it follows that, at frequencies $0<\omega<\omega_{b}(H)$, IGW amplitude attenuates with propagation distance $L$ as $\exp [-L I m$ $(\delta k)]$ due to continuous radiation of mechanical energy into the atmosphere. Since $R \ll 1$, an IGW needs to propagate a long distance of the order of $R^{-1}$ of its wavelengths to radiate half of its energy into the atmosphere. As a function of time $t$, the energy $E$ of free IGWs decreases exponentially: $E(t)=E(0) \exp (-t / T)$, where the $e$ folding time $T=\left[2 c_{g} \operatorname{Im}(\delta k)\right]^{-1}$ is usually referred to as the decay time. From Equations (1) and (7), for the IGW group speed $c_{g}$ and decay time, we find

$$
c_{g}=\left(\frac{\partial \omega}{\partial k}\right)_{H}=\frac{\omega}{k} \cdot \frac{2 k H+\sinh 2 k H}{2 \sinh 2 k H}
$$

and

$$
T=\frac{g\left(k^{2}-\omega^{2} c_{s}^{-2}\right)}{R \omega^{3}|\operatorname{Re}(m)|} .
$$

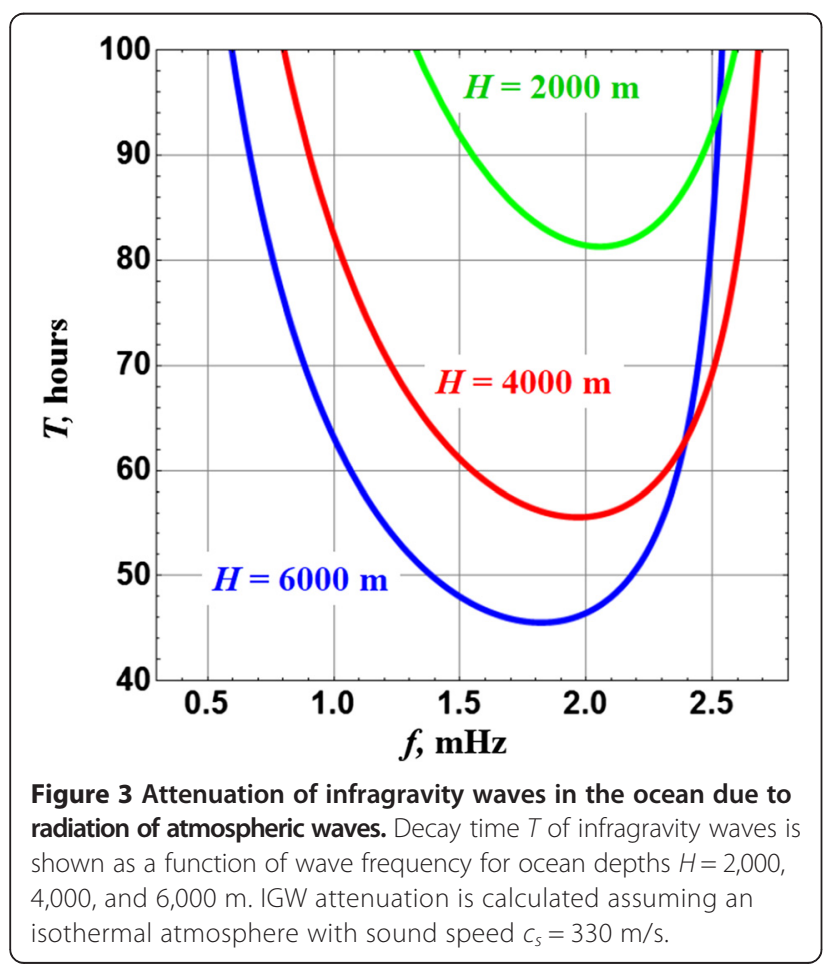

Here, $k$ is the real-valued IGW wavenumber; small relative corrections of the order of $R$ are neglected in Equations (7) and (10). IGW attenuation due to AGW radiation is small over the wave period, and the decay time is usually large since the air-to-water density ratio $R \ll 1$ (Figure 3). As expected, the decay time tends to infinity at the transition frequency $\omega_{b}$, where $m=0$.

In addition to radiation of atmospheric waves, which takes place only at frequencies $\omega<\omega_{b}(H)$, many other processes (viscous attenuation, bottom friction and percolation, scattering at small-scale bathymetric features, nonlinear interaction with other ocean wave types) can potentially contribute to IGW attenuation. Energy loss at reflection from coastlines is usually considered to be the dominant decay mechanism, at least for long-period IGWs such as tsunamis (Munk et al. 1962; Munk 1963; Van Dorn 1984, 1987). Recent measurements by Rabinovich et al. (2013) give decay rates of 18.5 to $20.0 \mathrm{~h}$ and 20.5 to $21.5 \mathrm{~h}$ for the 2010 Chile tsunami at wave periods 6 to $20 \mathrm{~min}$ (wave frequency 0.8 to $2.8 \mathrm{mHz}$ ) and 20 to $60 \mathrm{~min}$ (wave frequency 0.3 to $0.8 \mathrm{mHz}$ ), respectively. For the 2011 Tohoku tsunami, the measured decay rates (Rabinovich et al. 2013) are 17.0 to $19.0 \mathrm{~h}$ and 21.3 to $25.0 \mathrm{~h}$ at wave periods 6 to $20 \mathrm{~min}$ and 20 to $60 \mathrm{~min}$, respectively. Similar decay rates were derived previously by Munk (1963) and Van Dorn (1984) from much scarcer data sets than those employed in the recent study by Rabinovich et al. (2013).

Comparison of the predicted contribution of the atmospheric wave radiation (Figure 3) and measured (Munk 1963; Van Dorn 1984; Rabinovich et al. 2013) IGW decay rates shows that (i) IGW energy loss due to AGW radiation is consistent with available experimental data; (ii) radiation of atmospheric waves is a nonnegligible mechanism of attenuation of IGWs away from shore; and (iii) depending on the average ocean depth, AGW radiation can account for up to one third of the observed decay of IGWs with periods from 6 to $20 \mathrm{~min}$.

\section{Experiment Methods}

We use Dynasonde techniques to measure spectra of vertical velocity disturbances in the upper atmosphere. Dynasonde is a method of precision ionospheric radio sounding, which is based on detection of radio echoes, i.e., radio wave reflections from the inhomogeneous ionospheric plasma, in a broad frequency range of 1 to $20 \mathrm{MHz}$. Various analysis routines specific for Dynasonde applications perform inversions of measured parameters (range, angles of arrival, polarization, line-ofsight Doppler speed, amplitude) of the echoes to retrieve a number of ionospheric parameters (vertical profile of the plasma density, vector velocities of the plasma structures, spectra of small-scale irregularities, etc.) attributed to real 

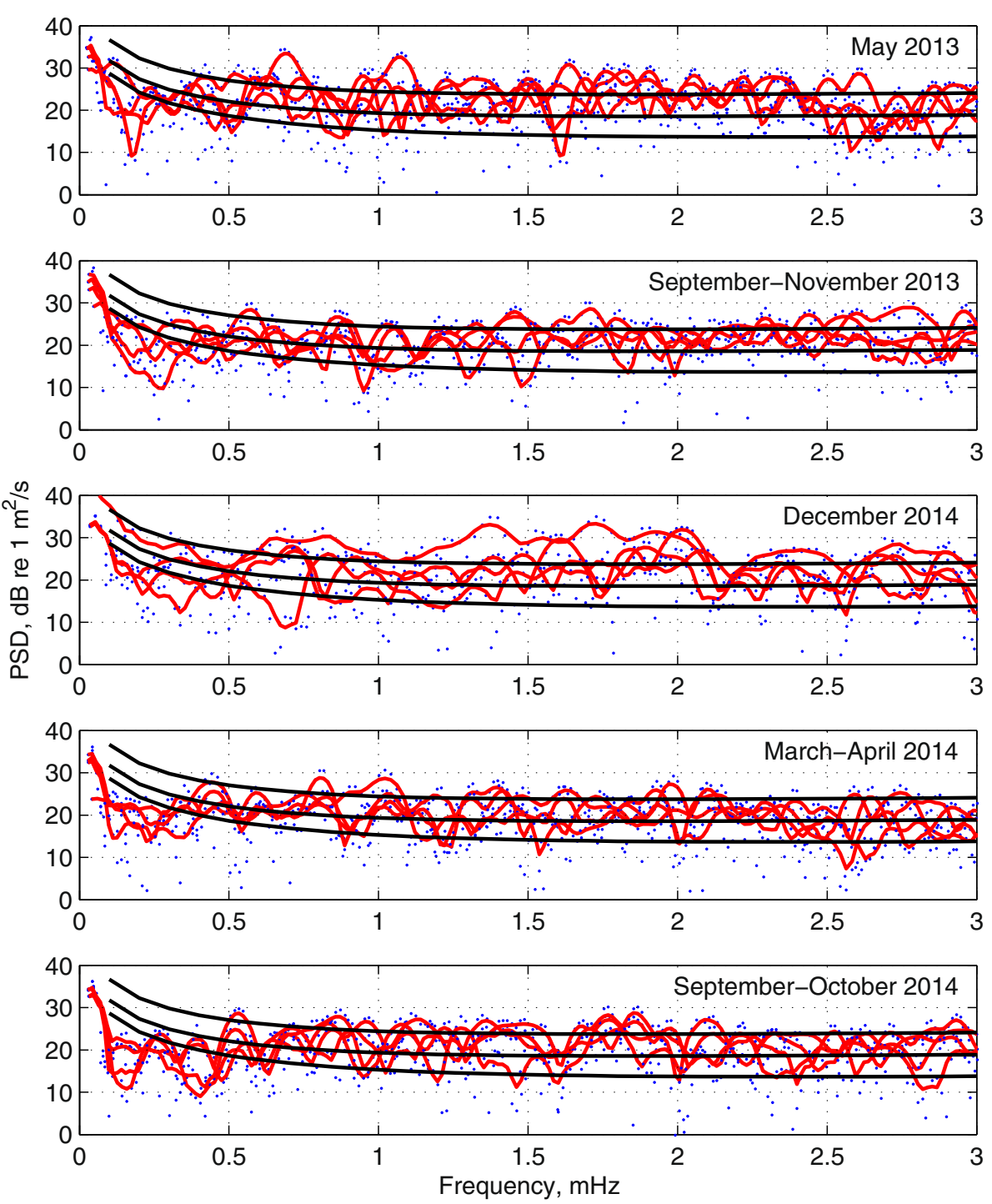

Figure 4 Measured power spectra. Measured power spectra of velocities' fluctuations along the magnetic field line at an altitude of $150 \mathrm{~km}$ are compared with predicted contributions due to atmospheric waves radiated by infragravity waves in the ocean. The spectra are measured by radio sounding of the ionosphere using an onshore Dynasonde (Wright and Pitteway 1999; Zabotin et al. 2006) HF radar system located at NASA's Wallops Island Flight Facility, Virginia, USA. The five panels refer to five distinct seasons in 2013 to 2014, and each panel contains four experimental spectral estimates obtained from the data series, specific dates and times of which are listed in the text. Blue dots show the results of spectral measurements. Red curves represent the results of smoothing the experimental spectra using a $0.5-\mathrm{mHz}$ sliding window. Black curves show contributions of the atmospheric waves of oceanic origin, which are estimated for various ocean depths (200, 1,000, and 5,000 m, top to bottom) using a semi-empirical model (Godin et al. 2013) of the IGW spectra.

altitudes (Wright and Pitteway 1999; Zabotin et al. 2006). The transition from the echo ranges measured in the socalled virtual space (where the difference between the group speed of the high-frequency (HF) electromagnetic waves in plasma and the vacuum speed of light is neglected) to actual spatial coordinates is essential for accuracy of our analysis. A component of Dynasonde data analysis software, the 3-D plasma density inversion procedure NeXtYZ (Zabotin et al. 2006), finds 3-D spatial location of the reflection point of each echo. The inversion procedure possesses the accuracy and sensitivity, which are necessary to visualize and measure wave activity in the upper atmosphere. Averaging of the vertical component of the Doppler velocities for all echoes reflected within a narrow altitude range during a sounding session produces a single data point for our analysis.

We have used data obtained with the Wallops Island VIPIR/Dynasonde HF radar system in 2013 to 2014 in the regime where the sounding sessions were repeated every $2 \mathrm{~min}$. The data screening has been performed 
using web-based visualization tools both provided by the standard Dynasonde software suite (Zabotin et al. 2005) and developed specifically for this project. Figure 5 presents an example of the standard output of the visualization software: a day-long scan of the vertical Doppler speed values shown by color as a function of the time of the day and of the real altitude. One can see a typical transition from night to day conditions near 13 UT and a transition from day to night conditions near 21 UT. The slightly inclined color strips are indications of the traveling ionospheric disturbances with their phase fronts propagating downward and therefore most likely caused by the acoustic-gravity waves propagating upward. Several criteria were used for the data selection. First of all, errors in ionogram trace identification and screening made by autonomous analysis must be infrequent in the data sets to not influence the statistics. Second, data series used for the spectral calculations must be long enough (at least several hours) because the frequencies of the wave disturbances of interest are as low as $0.1 \mathrm{mHz}$. We have adopted the coupling model between ionized and neutral components of the atmosphere according to which the velocity of the ion motion is estimated as a projection of the AGW-induced velocity perturbations onto the magnetic field line direction (Nicolls et al. 2014). This means that our analysis requires data from the upper ionosphere (above the $\mathrm{E}$ region). On the other hand, we cannot go too high because we would like to minimize the uncertainties and complications associated with the AGW absorption due to air viscosity and thermal conductivity. Only daytime ionospheric sounding data can provide information about the desired 130- to $180-\mathrm{km}$ altitude range. Ionospheric reflections from the altitudes below $150 \mathrm{~km}$ are often blanketed by the underlying regular and sporadic $\mathrm{E}$ layers. The gray lacuna in Figure 5 between approximately 14 and 20 UT and between roughly 110 and 140 $\mathrm{km}$ is an example of manifestation of such blanketing. One can tolerate infrequently missing data points due to, for example, occasional high radio interference at some narrow frequency bands or due to short-lasting sporadic layers. But long interruptions in data series should be avoided. That is why summer seasons, when distortions to the data caused by sporadic E layers are common, are not suitable for this analysis.

Combination of all the factors stated above makes $150 \mathrm{~km}$ the optimal altitude for data collection. Specifically, the following 20 time intervals, each one between 6 and $11 \mathrm{~h}$ long, were selected: 12 to 23 UT on 8 May 2013, 11 to 21 UT on 9 May 2013, 12 to 22 UT on 14 May 2013, 11 to 23 UT on 18 May 2013, 12 to 22 UT on 29 September 2013, 12 to 21 UT on 24 October 2013, 14 to 20 UT on 17 November 2013, 13 to 22 UT on 30 November 2013, 13 to 21 UT on 15 December 2013, 13 to 21 UT on 22 December 2013, 13 to 21 UT on 27 December 2013, 13 to 21 UT on 29 December 2013, 12 to 22 UT on 10 January 2014, 12 to 22 UT on

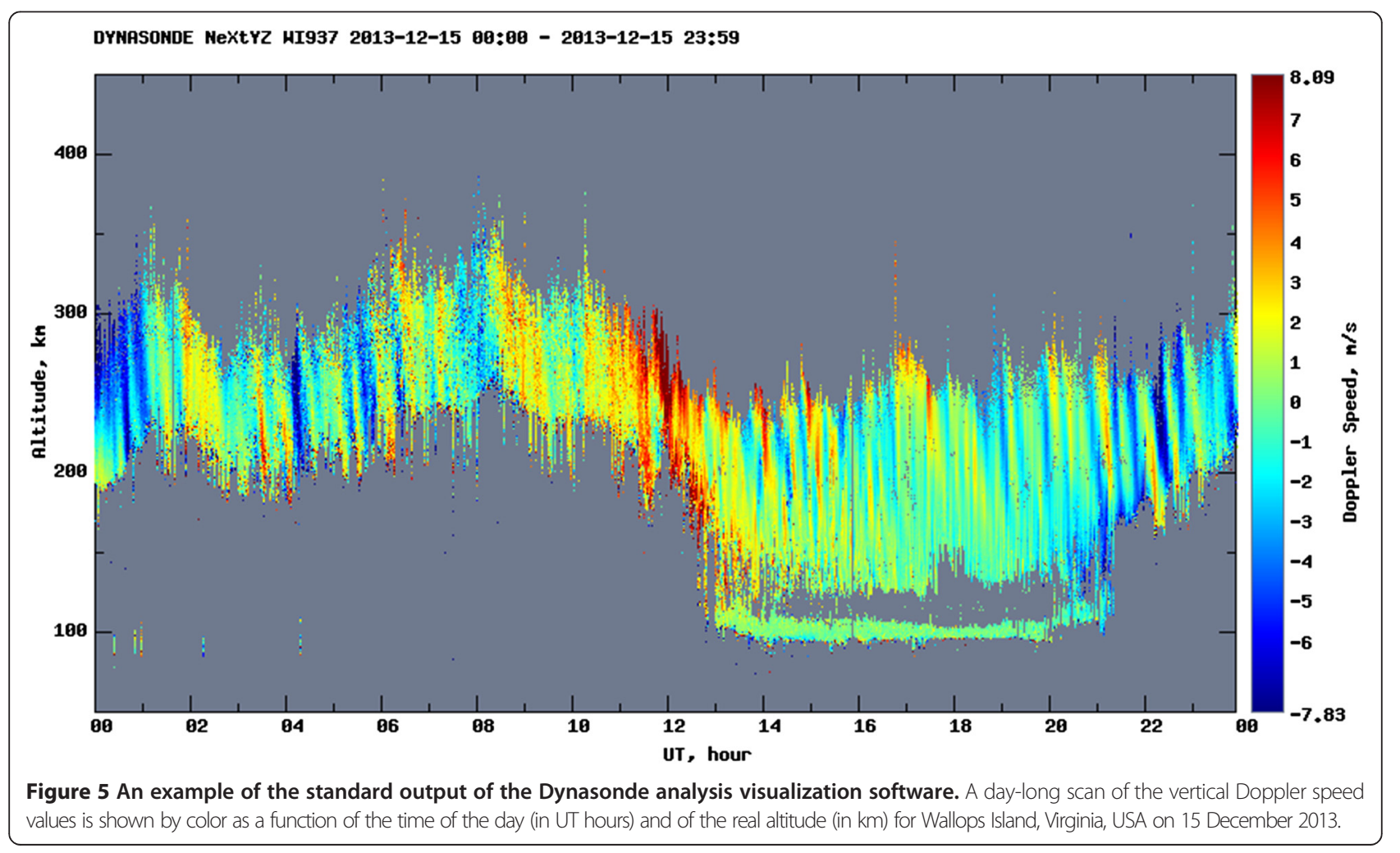


15 March 2014, 12 to 22 UT on 18 March 2014, 12 to 22 UT on 22 April 2014, 13 to 20 UT on 26 April 2014,12 to 21 UT on 26 September 2014, 13 to 22 UT on 29 September 2014, and 12 to 22 UT on 30 September 2014.

Every data point was obtained by averaging the vertical projections of the line-of-sight Doppler speed data for the 150 - to $151-\mathrm{km}$ altitude range. All the time intervals are affected by the diurnal variations of the Doppler speed; there is a background motion of the ionization layers downward after sunrise and upward before sunset. To suppress the spectral harmonics related to the diurnal variations, the Hann window was used for every data segment, with subsequent application of restoration coefficient 2 to the resulting power spectra. Some of the data points are missing, because of the reasons stated above, so the data series are inherently non-uniform. To account for this, the spectrum evaluation was performed with Hocke's (1998) modification of the Lomb-Scargle algorithm.

The experimental Doppler speed spectra were converted into the spectra of the ion movements by applying the factor $\sin ^{-2} \phi$, where $\phi$ is the magnetic dip angle. These are compared to the power spectra of the IGWinduced velocities parallel to the magnetic field line at an altitude of $150 \mathrm{~km}$, which are calculated using the semi-empirical model spectra $S(\omega, H)$ (Godin et al. 2013) of the ocean surface displacements caused by the infragravity waves. The altitude chosen for the comparison is low enough (Hines 1960) to neglect effects of absorption of IGW-radiated acoustic-gravity waves, which have frequencies below approximately $3 \mathrm{mHz}$ and horizontal spatial scales of tens and hundreds of kilometers. From AGW polarization relations (e.g., Godin 2012), we find the relation

$$
\left\langle v_{m}^{2}\right\rangle=\left\langle v_{3}^{2}\right\rangle\left[\sin ^{2} \varphi+\frac{(\gamma-1) g^{2} \omega^{-2}-c_{s}^{2}}{2\left(c_{s}^{2}-\omega^{2} k^{-2}\right)} \cos ^{2} \varphi\right]
$$

between the wave-induced fluctuations $v_{m}$ of the velocity along the magnetic line and the vertical velocity $v_{3}$ in a stationary atmosphere. Here, angular brackets denote statistical averaging. The first and second terms in the brackets in Equation (11) come from the wave-induced vertical and horizontal motions, respectively. We assume here that the IGW field is isotropic. The power spectrum $S_{v}(\omega, z)$ of the vertical velocity fluctuations $v_{3}$ at height $z$ in the atmosphere is related to the spectrum of the ocean surface elevations as follows: $S_{v}(\omega, z)=\omega^{2}$ $\left[\rho_{a}(0) / \rho_{a}(z)\right] S(\omega, H)$. The power spectra of the IGWinduced fluctuations $v_{m}$ of the velocity along the magnetic line, which are calculated from $S_{v}(\omega, z)$ using Equation (11), are utilized below for comparisons with experimental $v_{m}$ power spectra that are derived from Doppler speed measurements (Figure 4). Note that in a vertically stratified atmosphere with the horizontal winds, the total vertical power flux carried by an acoustic-gravity wave remains unchanged until the wave reaches a turning point, and existence and altitude of the turning points depend on the direction of wave propagation in the horizontal plane (Fritts and Alexander 2003). Therefore, the details of the vertical stratification are much less significant for the integral quantities considered in this paper than, for example, for calculating the spatial distribution of the wave field.

\section{Observations}

Atmospheric waves are known to be generated by a number of physical processes, associated most notably with convection, wind shear, and air-flow interaction with orography (Fritts and Alexander 2003; Geller et al. 2013). To assess the significance of the IGW contribution to the atmospheric wave field in the middle and upper atmosphere above oceans, we compare statistics of measured fluctuations of velocity with the velocity fluctuations due to random AGWs radiated by background IGWs in the ocean (Figure 4). To our knowledge, detailed measurements of velocity fluctuations in the middle and/or upper atmosphere above the ocean are not available at this time. As a proxy for the atmosphere above the ocean, we use experimental results obtained with HF radar systems at a coastal location in the North Atlantic.

Specifically, we use the data obtained with the VIPIR/ Dynasonde HF radar system located at NASA's Wallops Flight Facility in Virginia, USA. Projections of the velocity fluctuations onto the magnetic field line direction at ionospheric altitudes and their power spectra, which are shown in Figure 4, are retrieved from Doppler shifts of radio echoes reflected by the ionosphere in the real altitude range of 150 to $151 \mathrm{~km}$ (Wright and Pitteway 1999). The choice of this altitude range is determined by specifics of the ionospheric radio sounding as explained in the previous section. Figure $2 \mathrm{~d}$ shows that AGWs of oceanic origin propagate obliquely away from the ocean surface. AGWs observed by the radar would be radiated by free IGWs at different distances from shore depending on the wave frequency and bathymetry. For comparison with the measured spectra, in Figure 4, we present predictions for oceans with three different depths $H$, which encompass the range of the relevant $H$ values in the vicinity of Wallops. The IGW contribution to velocity fluctuations in the atmosphere is expected to be a weighted average of spectra corresponding to different $H$.

Although measured velocity fluctuations include contributions of both atmospheric waves of various origins and turbulence, Figure 4 shows that the expected velocity fluctuations due to the waves of oceanic origin are within the range covered by the natural variability of the 
measured spectra. Comparison of the smoothed measured spectra (red lines in Figure 4) and theoretical predictions (black lines in Figure 4) indicates that there are extended periods of time when the bulk of the velocity variance at the thermospheric altitudes observed at Wallops can be attributed to AGWs of oceanic origin. These observations suggest the feasibility of direct measurements that would confirm correlation (coherence) between the wave phenomena in both media. A project of this kind is underway with a support from the Basic Research Challenge program of the US Office of Naval Research (Zabotin et al. 2013).

From tropospheric to mesospheric heights, wave processes are believed to be dominated by AGWs that are generated by moist convection, shear flow instabilities, jets, atmospheric fronts, and interaction of winds with the earth's topography (Gossard and Hooke 1975; Fritts and Alexander 2003; Geller et al. 2013). The power flux in these AGWs rapidly decreases with height due to viscous dissipation, wave breaking, and blocking at the critical levels (Gossard and Hooke 1975; Fritts and Alexander 2003; Geller et al. 2013). Only a very small (and unknown) fraction of the energy of these AGWs reaches ionospheric heights. AGWs of oceanic origin, and especially the AGWs radiated by IGWs in deep water, are characterized by considerably higher horizontal phase speeds, which can exceed the maximum wind speeds. This leads to larger horizontal spatial scales and, correspondingly, to weaker viscous absorption (Godin 2014). Fast AGWs do not have critical levels and escape the critical level filtering (Hines 1960; Gossard and Hooke 1975). Therefore, it should not be surprising that the AGWs of oceanic origin, which have small amplitudes and are hardly observable in the troposphere and stratosphere, play a much larger role at ionospheric heights.

\section{Conclusion}

Atmospheric loading leads to a qualitative change in properties of infragravity waves in the ocean. These waves cease to be surface ones and become leaky waves at frequencies below a certain depth-dependent transition frequency of the order of $3 \mathrm{mHz}$. The leaky waves continuously radiate a small fraction of their energy into the atmosphere as acoustic-gravity waves. The acousticgravity waves of oceanic origin propagate into the middle and upper atmosphere, where they appear to account for a significant part of the observed velocity fluctuations. As the atmospheric waves dissipate at high altitudes, they impact the atmospheric dynamics through deposition of mechanical energy and momentum transported from the ocean. Further research is needed to refine the simple model presented in this paper by account of geographic and seasonal variability of IGWs in the ocean and three-dimensional inhomogeneity of the atmosphere, in order to quantify the energy and momentum transport due to radiation of long atmospheric waves by background infragravity waves.

\section{Abbreviations}

AGW: acoustic-gravity wave; IGW: infragravity wave; UT: universal time.

\section{Competing interests}

The authors declare that they have no competing interests.

\section{Authors' contributions}

OG and NZ conceived this project and wrote the paper. OG developed the analytical theory. NZ performed numerical calculations and Dynasonde data analysis. TB performed radar experiments and collected data. All authors read and approved the final manuscript.

\section{Acknowledgements}

The authors are grateful to L. Zabotina for development of web-based visualization software for results of the Dynasonde data analysis. Insightful comments by Nozomu Nishitani and two anonymous referees helped to improve presentation. The work reported in this paper was supported by the Office of Naval Research through award N00014-13-1-0348. This project utilized the Janus supercomputer, which is supported by the National Science Foundation (award number CNS-0821794) and operated by the University of Colorado Boulder. The VIPIR/Dynasonde system is hosted by NASA's Wallops Flight Facility.

\section{Author details}

${ }^{1}$ Cooperative Institute for Research in Environmental Sciences, University of Colorado, Boulder, CO 80309, USA. ²Physical Sciences Division, NOAA Earth System Research Laboratory, Boulder, CO 80305, USA. ${ }^{3}$ Department of Electrical, Computer, and Energy Engineering, University of Colorado, Boulder, CO 80309, USA. ${ }^{4}$ NOAA National Geophysical Data Center, Boulder, CO 80305, USA.

Received: 10 October 2014 Accepted: 15 March 2015

Published online: 02 April 2015

\section{References}

Artru J, Ducic V, Kanamori H, Lognonné P, Murakami M (2005) lonospheric detection of gravity waves induced by tsunamis. Geophys J Int 160:840-848

Aucan J, Ardhuin F (2013) Infragravity waves in the deep ocean: an upward revision. Geophys Res Lett 40:3435-3439

Brekhovskikh LM, Godin OA (1998) Acoustics of layered media. 1: plane and quasi-plane waves. Springer, Heidelberg

Bromirski PD, Stephen RA (2012) Response of the Ross Ice Shelf, Antarctica, to ocean gravity-wave forcing. Ann Glaciol 53:163-172

Crawford WC, Webb SC, Hildebrand JA (1998) Estimating shear velocities in the oceanic crust from compliance measurements by two-dimensional finite difference modeling. J Geophys Res 103:9895-9916

Eckart C (1953) The theory of noise in continuous media. J Acoust Soc Am 25:195-199

Fritts DC, Alexander MJ (2003) Gravity wave dynamics and effects in the middle atmosphere. Rev Geophys 41:1003

Galvan DA, Komjathy A, Hickey MP, Stephens P, Snively J, Song YT, Butala MD, Mannucci AJ (2012) lonospheric signatures of Tohoku-Oki tsunami of March 11, 2011: model comparisons near the epicenter. Radio Sci 47:RS4003

Garces MA, Perttu A, Williams B (2011) Infrasonic troposphere-ionosphere coupling in Hawaii, Paper SA23A-1897 presented at the 2011 AGU Fall Meeting, San Francisco, CA, USA, 5-9 December 2011

Geller MA, Alexander MJ, Love PT, Bacmeister J, Ern M, Hertzog A, Manzini E, Preusse P, Sato K, Scaife AA, Zhou T (2013) A comparison between gravity wave momentum fluxes in observations and climate models. J Clim 26:6383-6405

Gill AE (1982) Atmosphere-ocean dynamics. Academic, New York

Godin OA (2012) Acoustic-gravity waves in atmospheric and oceanic waveguides. J Acoust Soc Am 132:657-669

Godin OA (2014) Dissipation of acoustic-gravity waves: an asymptotic approach. J Acoust Soc Am 136:EL411-EL417

Godin OA, Fuks IM (2012) Transmission of acoustic-gravity waves through gas-liquid interfaces. J Fluid Mech 709:313-340 
Godin OA, Zabotin NA, Sheehan AF, Yang Z, Collins JA (2013) Power spectra of infragravity waves in a deep ocean. Geophys Res Lett 40:2159-2165

Godin OA, Zabotin NA, Sheehan AF, Collins JA (2014) Interferometry of infragravity waves off New Zealand. J Geophys Res Oceans 118:1103-1122

Gossard EE, Hooke WH (1975) Waves in the atmosphere. Elsevier, Amsterdam

Herbers THC, Elgar S, Guza RT (1995) Generation and propagation of infragravity waves. J Geophys Res 100:24863-24872

Hines CO (1960) Internal atmospheric gravity waves at ionospheric heights. Can J Phys 38:1441-1481

Hines CO (1972) Gravity waves in the atmosphere. Nature 239:73-78

Hocke K (1998) Phase estimation with the Lomb-Scargle periodogram method. Ann Geophys 16:356-358

Komjathy A, Galvan DA, Stephens P, Butala MD, Akopian V, Wilson VO, Mannucc AJ, Hickey M (2012) Detecting ionospheric TEC perturbations caused by natural hazards using a global network of GPS receivers: the Tohoku case study. Earth Planets Space 64:1287-1294

Livneh DJ, Seker I, Djuth FT, Mathews JD (2007) Continuous quasiperiodic thermospheric waves over Arecibo. J Geophys Res 112, A07313

Makela JJ, Lognonné $\mathrm{P}$, Hébert H, Gehrels T, Rolland L, Allgeyer S, Kherani A, Occhipinti G, Astafyeva E, Coïsson P, Loevenbruck A, Clévédé E, Kelley MC, Lamouroux J (2011) Imaging and modeling the ionospheric airglow response over Hawaii to the tsunami generated by the Tohoku earthquake of 11 March 2011. Geophys Res Lett 38:L13305

Munk WH (1963) Some comments regarding diffusion and absorption of tsunamis. In: Proceedings of the Tsunami Meeting, X Pacific Science Congress, IUGG Monographs, 24th edn. IUGG, Paris, pp 53-72

Munk WH, Miller GR, Snodgrass FE (1962) Long-period waves over California's continental borderland, III. The decay of tsunamis and the dissipation of tides. J Mar Res 20:119-120

Nicolls MJ, Vadas SL, Aponte N, Sulzer MP (2014) Horizontal parameters of daytime thermospheric gravity waves and E region neutral winds over Puerto Rico. J Geophys Res 119:575-600

Occhipinti G, Rolland L, Lognonné P, Watada S (2013) From Sumatra 2004 to Tohoku-Oki 2011: the systematic GPS detection of the ionospheric signature induced by tsunamigenic earthquakes. J Geophys Res 119:3626-3636

Peltier WR, Hines CO (1976) On the possible detection of tsunamis by monitoring of the ionosphere. J Geophys Res 81:1995-2000

Rabinovich AB, Candella RN, Thomson RE (2013) The open ocean energy decay of three recent trans-Pacific tsunamis. Geophys Res Lett 40:3157-3162

Rhie J, Romanowicz B (2004) Excitation of Earth's continuous free oscillations by atmosphere-ocean-seafloor coupling. Nature 431:552-556

Tsai VC, Ampuero J-P, Kanamori H, Stevenson DJ (2013) Estimating the effect of Earth elasticity and variable water density on tsunami speeds. Geophys Res Lett 40:492-496

Van Dorn WG (1984) Some tsunami characteristics deducible from tide records. J Phys Oceanogr 14:353-363

Van Dorn WG (1987) Tide gage response to tsunamis. Part II: other oceans and smaller seas. J Phys Oceanogr 17:1507-1516

Watada S, Kusumoto S, Satake K (2014) Traveltime delay and initial phase reversal of distant tsunamis coupled with the self-gravitating elastic Earth. J Geophys Res 119:4287-4310

Webb SC, Zhang X, Crawford W (1991) Infragravity waves in the deep ocean. J Geophys Res 96:2723-2736

Wright JW, Pitteway MLV (1999) A new data acquisition concept for digital ionosondes: phase-based echo recognition and real-time parameter estimation. Radio Sci 34:871-882

Zabotin NA, Wright JW, Bullett TW, Ye Zabotina L (2005) Dynasonde 21 principles of data processing, transmission, storage and web service. In: Proceedings of the ionospheric effects symposium 2005. Alexandria, VA, USA., pp 7B3-1-7B3-3

Zabotin NA, Wright JW, Zhbankov GA (2006) NeXtYZ: three-dimensional electron density inversion for dynasonde ionograms. Radio Sci 41:RS6S32

Zabotin NA, Godin OA, Bullet TW (2013) Tracing acoustic-gravity waves from the ocean into the ionosphere: ONR BRC Project., University of Colorado Boulder. http://surf.colorado.edu. Accessed 29 Dec 2014

\section{Submit your manuscript to a SpringerOpen ${ }^{\circ}$ journal and benefit from:}

- Convenient online submission

- Rigorous peer review

- Immediate publication on acceptance

- Open access: articles freely available online

- High visibility within the field

- Retaining the copyright to your article

Submit your next manuscript at $>$ springeropen.com 